\title{
PERSPECTIVAS SOBRE EDUCACIÓN EN VALORES EN TIEMPOS DE CRISIS
}

\section{APPROACHES ON VALUES EDUCATION IN TIMES OF CRISIS}

\author{
Gladys Merma Molina \\ Salvador Peiró i Gregòri \\ Diego Gavilán Martín \\ Universidad de Alicante. España/Spain \\ gladys.merma@ua.es \\ salvador.peiro@ua.es \\ diego.gavilan@ua.es
}

Recibido/Received: 07/02/2013

Modificado/Modified: 12/03/2013

Aceptado/Accepted: 06/05/2013

\section{RESUMEN}

En medio de la actual crisis económica mundial, todas las instituciones de la sociedad están afectadas por la crisis moral, de manera que existe una evidente y preocupante contradicción entre ellas. Esta crisis moral implica la carencia de valores personales y ciudadanos, que afectan, significativamente, al desarrollo del individuo y a la convivencia social. Tratamos de verificar si hay indicios de ello en la realidad escolar con relación a la axiología curricular y su desarrollo. Para analizar la situación de la enseñanza-aprendizaje de los valores en la educación española, hemos aplicado una encuesta a 1320 profesores de educación secundaria, con el fin de averiguar los objetivos, los tipos de valores y las habilidades personales y sociales, que desarrollan en el aula. La situación nos indica que son necesarios nuevos enfoques de educación en valores, que se adapten a estos tiempos.

La formación axiológica debe estar incorporada en todos los ámbitos del conocimiento y ha de ir a la par que la formación de la inteligencia. El binomio actividad intelectual-actividad moral ha de formar un todo insociable. En esta propuesta, reflexionamos en torno a la aplicación de un nuevo paradigma axiológico que se separe del racionalismo mecanicista, y que se oriente a una educación personal holística, sistemática, interdisciplinaria y transversal a todo el currículo educativo.

\section{PALABRAS CLAVE}

Educación en valores, crisis económica, valores éticos, valores universales, escuela.

\section{SUMARIO}

1. Situación actual de la educación en valores. 2. Qué y cómo educar en valores, en tiempos de crisis. 3. Conclusiones e implicaciones. Bibliografía

\footnotetext{
ABSTRACT

Amid the current global economic crisis, all institutions of society are affected by the moral crisis, so there is a clear and disturbing contradiction between them. This moral crisis involves the lack of personal values and citizens, affecting, significantly, the development of individual and social life. We try to check for signs of it in the axiological dimension of schools regarding curriculum and its development. To analyze the situation of the Spanish educational system about learning values, we
} 
applied a 1320 survey of high school teachers, in order to determine the objectives, the types of values and personal and social skills that are developed in the classroom. The situation indicates that new approaches are needed to value education that fit these times. Axiological training should be incorporated into all areas of knowledge and must be matched to the training of intelligence. The binomial intellectual activity and moral activity must form a whole unsociable. In this proposal we reflect on the implementation of a new paradigm that separates axiological mechanistic rationalism, and aimed at a holistic axiological education, systematic, interdisciplinary and cross the entire curriculum.

\section{KEYWORDS}

Values education, economic crisis, ethical values, universal values, school.

\section{CONTENTS}

1. Situation present of values education. 2. What and how to teach values, in times of crisis. 3 . Conclusions and implications. References.

\section{LA SITUACIÓN AXIOLÓGICA ESCOLAR ACTUAL}

En nuestra sociedad postmoderna se confunden los valores y antivalores. Hoy conviven amenazas de guerra y destrucción con movimientos carismáticos, escándalos de corrupción de todo tipo y en todos los niveles, bandas de terrorismo juveniles con organizaciones de jóvenes comprometidos por la lucha contra la injusticia y la defensa de los más pobres.

Los casos de corrupción, que han ocurrido en el entorno político, a nivel nacional e internacional, brindan una nueva imagen de lo que, en la actualidad, se entiende por "democracia". Aún más grave es la violencia, que cada vez se hace evidente, en nuestra sociedad. Por ejemplo, la matanza perpetrada en Oslo (Noruega), en 2011; el asesinato de 27 personas, 20 de ellos niños, en Connecticut (EE.UU.), en 2012, o los disturbios ocurridos, últimamente, en algunas ciudades europeas, americanas e inglesas, son el reflejo de una sociedad convulsa y carente de valores. Es notorio el "colapso moral". Indudablemente, la crisis económica global está asociada a una crisis moral global (Merma, 2011).

Con todo este panorama, queda demostrado que hechos como los que hemos señalado no sólo ocurren en los países pobres, sino también en aquellos que tienen un alto desarrollo económico y nivel democrático. Hay un sustrato de violencia social, de exclusión, de racismo y de desequilibrio de principios y valores, en todas las sociedades, donde lo único que importa es el crecimiento económico. El incremento de la desigualdad y de la brecha social agrava la crisis. La crisis económica ha provocado también un profundo declive de las grandes creencias y filosofías. Constantemente, se habla del déficit, del desempleo, de la economía sumergida, etc., pero no de los problemas morales que todo esto conlleva. Los jóvenes necesitan encontrar un nuevo proyecto que dé sentido a su presente y que les oriente hacia el futuro.

Gran parte de contextos, grupos e instituciones de la sociedad (familia, escuela, medios de comunicación, grupos políticos y de poder, etc.) están afectados por esta crisis de valores, de manera que existe una evidente y preocupante contradicción entre ellas (López-Barajas, 2012). La crisis moral implica la carencia de valores tanto personales como ciudadanos, que afectan, significativamente, al desarrollo del individuo y a la convivencia social.

En el contexto de la teoría axiológica más pura (formalismo kantiano), sabemos que aunque los valores sean "entidades objetivas" o "cualidades abstractas", estos están 
vinculados con la conciencia y con la capacidad valorativa del ser humano, y si bien existen unos valores universales absolutos, el contexto socio-cultural será determinante.

Las jerarquías valorativas cambian y fluctúan de acuerdo al contexto (Benbenishty y Astor, 2005). Sin dejar de mencionar a las teorías subjetivistas, que sostienen que los valores se hallan en la vida humana y son determinados en su ser y su jerarquía, y a las objetivistas, que afirman que los valores son simplemente descubiertos por el hombre, la discusión sobre la jerarquía de valores nos lleva a plantear, sin caer en el absolutismo axiológico, que, en cualquier caso, existen unos valores y unos principios éticos universales, aceptados por toda la humanidad, que van más de creencias, doctrinas y cosmovisiones particulares del mundo. Y que tales realidades son ideas-fuerza que relacionan a los sujetos entre sí, a la vez que las descubrimos en los bienes de la cultura y las conductas de nuestros semejantes.

En consecuencia, esta categoría de valores éticos y morales universales (libertad, solidaridad, cooperación, respeto, paz, igualdad, etc.) deben ser incorporados, como una parte integrante del currículo educativo, sin menoscabar otros valores como los intelectuales (sabiduría), afectivos (amor, afecto), sociales (interacción, comunicación, adaptación, sensibilidad social) y, por supuesto, personales (autoestima, autocontrol).

La formación axiológica debe estar incorporada en todos los ámbitos del conocimiento transversalidad de los valores en el currículo-, y ha de ir a la par que la formación de la inteligencia. El binomio actividad intelectual-actividad moral, a la que ya había hecho referencia el constructivismo de Piaget, ha de formar un todo insociable.

La "realidad pedagógica" de los valores se muestra explícitamente en los resultados que hemos obtenido a partir de una encuesta aplicada a 1320 profesores de educación secundaria, en la Comunidad Valenciana (España) (Merma, 2011). Con relación a los objetivos que se deben lograr con la educación en valores, es mayoritario el porcentaje de profesores $(100 \%$, en 2009-2010 y 72,88\%, en 2010-2011) que afirman que su objetivo principal, al enseñar valores, es motivar al alumno para que piense y decida por sí mismo (Tabla $\mathrm{N}^{\mathrm{o}} 1$ ).

Tabla 1. Objetivos de la educación en valores

\begin{tabular}{|l|c|c|c|}
\hline & PROF. 2009-2010 & PROF. 2010-2011 & VARIACIÓN \\
\hline Inculcar valores para ser bueno & $12,50 \%$ & $33,90 \%$ & $21,40 \%$ \\
\hline Motivarlo para que piense y decida por sí mismo. & $100,00 \%$ & $72,88 \%$ & $-27,12 \%$ \\
\hline Incidir para que cambie su comportamiento & $50,00 \%$ & $27,12 \%$ & $-22,88 \%$ \\
\hline Mejorar las normas del aula. & $37,50 \%$ & $49,15 \%$ & $11,65 \%$ \\
\hline Conseguir mayor disciplina y orden & $50,00 \%$ & $37,29 \%$ & $-12,71 \%$ \\
\hline Otras & $0,00 \%$ & $3,39 \%$ & $3,39 \%$ \\
\hline
\end{tabular}

Observamos que el objetivo más importante por los que se enseña valores, según los profesores entrevistados (para que piense y decida por sí mismo) ha disminuido notablemente del curso académico 2009 al curso académico 2010 (variación de -27,12\%). El segundo objetivo que les interesa es conseguir una mayor disciplina y orden para poder trabajar $(50,00 \%$, en 2009-2010) y mejorar el cumplimiento de normas dentro del aula (49,15\%, en el curso 2010-2011). Estos dos últimos datos están directamente relacionados con el incremento de la conflictividad escolar (situaciones en los que uno o varios alumnos impiden el desarrollo de la clase) y de la indisciplina en las aulas (conductas que van en contra de las normas del centro y del aula), que, en la actualidad, son los problemas que más afectan a los profesores, en España (Peiró, 2009). 
Por otra parte, sabemos que se deben ejercitar, permanentemente, buenas prácticas en el aula, basadas en la atención a la diversidad, mejora de comunicación, el respeto, la solidaridad y la cooperación. Para que estas estrategias se apliquen, es importante que el profesor esté respaldado por la dirección de su centro y por sus propios compañeros -de ahí la importancia de la comunicación y el trabajo en equipos-. Y, para ello hacen falta valores.

Por esto, definiendo aún más en nuestro diagnóstico, quisimos averiguar qué tipo de valores trabaja el profesorado. Los resultados se muestran en la Tabla $\mathrm{N}^{\circ} 2$.

Tabla 2: Tipos de valores

\begin{tabular}{|l|c|c|c|}
\hline & PROF. 2009-2010 & PROF. 2010-2011 & VARIACIÓN \\
\hline Autoconocimiento. & $62,50 \%$ & $35,59 \%$ & $-26,91 \%$ \\
\hline Relación entre compañeros. & $100,00 \%$ & $77,97 \%$ & $-22,03 \%$ \\
\hline Relaciones en la familia. & $62,50 \%$ & $52,54 \%$ & $-9,96 \%$ \\
\hline La discriminación por razón de género. & $37,50 \%$ & $50,85 \%$ & $13,35 \%$ \\
\hline La convivencia en la escuela. & $75,00 \%$ & $59,32 \%$ & $-15,68 \%$ \\
\hline Habilidades para el diálogo. & $75,00 \%$ & $35,59 \%$ & $-39,41 \%$ \\
\hline Avances científicos y tecnológicos. & $25,00 \%$ & $20,34 \%$ & $-4,66 \%$ \\
\hline Problemática ecológica. & $50,00 \%$ & $52,54 \%$ & $2,54 \%$ \\
\hline La diversidad del alumnado. & $75,00 \%$ & $50,85 \%$ & $-24,15 \%$ \\
\hline Los Derechos Humanos. & $62,50 \%$ & $62,71 \%$ & $0,21 \%$ \\
\hline El civismo. & $62,50 \%$ & $37,29 \%$ & $-25,21 \%$ \\
\hline Los valores que marca la Ley de Educación. & $12,50 \%$ & $23,73 \%$ & $11,23 \%$ \\
\hline Valores constitucionales. & $12,50 \%$ & $25,42 \%$ & $12,92 \%$ \\
\hline Otros & $0,00 \%$ & $5,08 \%$ & $5,08 \%$ \\
\hline
\end{tabular}

Los resultados nos muestran que las situaciones que más se trabajan en el centro y en el aula son, en primer lugar, la relación entre compañeros (100\% y 77,97\%, en 2009-2010 y 2010-2011, respectivamente). Ocupan el segundo lugar la convivencia en la escuela, las habilidades de diálogo y la diversidad del alumnado (75,00\%), en el curso 2009-2010, y los Derechos Humanos (62,71\%), en el curso 2010-2011.

Por otro lado, un dato curioso es el hecho de que los valores que señala la Ley de Educación hayan obtenido porcentajes muy bajos (12,50\% y 25,42\%, en 2009-2010 y 20102011, respectivamente), en ambos cursos académicos. Esto puede ser debido a que, probablemente, los profesores desconozcan el contenido de dicha norma, con relación al tema que nos ocupa (Merma, 2012). Los valores, en la Ley Orgánica de Educación (2006), ya aparecen en el Artículo 1, cuando se habla de los Principios y fines de la Educación.

Otro dato que hay que destacar es el desarrollo de habilidades de diálogo. Este ítem es el que más ha variado negativamente $(-39,41)$. Las destrezas de comunicación son cruciales, ya que le permite al individuo relacionarse con otras personas y desenvolverse adecuadamente, y de manera eficaz, en su sociedad. Por ello, es importante trabajar el desarrollo de estas habilidades, desde su formación inicial. Asimismo, observamos que muchos otros valores importantes también han disminuido, negativamente, de un curso académico a otro. Este es el caso de las habilidades de diálogo $(-39,41 \%)$, autoconocimiento $(-26,91 \%)$, el civismo ($25,21 \%)$, la diversidad del alumnado $(-24,15 \%)$, relación entre compañeros $(-22,03 \%)$. La Educación en valores significa contribuir a que las personas acepten su cultura y se conozcan, primero a sí mismos, para que luego acepten a los demás, y sean competentes como ciudadanos de una sociedad, que se caracteriza por la diversidad.

Finalmente, quisimos saber el grado importancia que le daba el profesor al desarrollo de otras habilidades (capacidad para realizar determinadas actividades y tareas, de manera 
eficiente), actitudes (disposición permanente del individuo para actuar de una forma) y valores (cualidad por la que una persona o cosa merece ser apreciada). En concreto, la pregunta que formulamos fue: ¿Qué peso tiene el desarrollo de las siguientes capacidades y habilidades en los alumnos? Los resultados fueron los siguientes:

Tabla 3: Valoración de capacidades y habilidades

\begin{tabular}{|c|c|c|c|}
\hline & PROF. 2009-2010 & PROF. 2010-2011 & VARIACIÓN \\
\hline Autoconocimiento & $87,50 \%$ & $55,93 \%$ & $-31,57 \%$ \\
\hline Autonomía y autorregulación & $75,00 \%$ & $49,15 \%$ & $-25,85 \%$ \\
\hline Capacidades de diálogo & $75,00 \%$ & $55,93 \%$ & $-19,07 \%$ \\
\hline Capacidad para transformar el entorno & $50,00 \%$ & $37,29 \%$ & $-12,71 \%$ \\
\hline Comprensión crítica & $75,00 \%$ & $49,15 \%$ & $-25,85 \%$ \\
\hline Empatía y perspectiva social & $87,50 \%$ & $52,54 \%$ & $-34,96 \%$ \\
\hline Habilidades sociales y convivenciales & $87,50 \%$ & $54,24 \%$ & $-33,26 \%$ \\
\hline Razonamiento moral & $50,00 \%$ & $50,85 \%$ & $0,85 \%$ \\
\hline Informarse & $75,00 \%$ & $45,76 \%$ & $-29,24 \%$ \\
\hline Comunicar & $62,50 \%$ & $52,54 \%$ & $-9,96 \%$ \\
\hline Anticipar & $50,00 \%$ & $32,20 \%$ & $-17,80 \%$ \\
\hline Inventar & $50,00 \%$ & $37,29 \%$ & $-12,71 \%$ \\
\hline Negociar & $75,00 \%$ & $40,68 \%$ & $-34,32 \%$ \\
\hline Decidir & $75,00 \%$ & $55,93 \%$ & $-19,07 \%$ \\
\hline Imaginar & $50,00 \%$ & $47,46 \%$ & $-2,54 \%$ \\
\hline Cooperar & $87,50 \%$ & $69,49 \%$ & $-18,01 \%$ \\
\hline Evaluar & $37,50 \%$ & $33,90 \%$ & $-3,60 \%$ \\
\hline Asumir riesgos & $37,50 \%$ & $37,29 \%$ & $-0,21 \%$ \\
\hline Afrontar la complejidad & $75,00 \%$ & $40,68 \%$ & $-34,32 \%$ \\
\hline Analizar necesidades & $75,00 \%$ & $30,51 \%$ & $-44,49 \%$ \\
\hline Llevar a cabo proyectos & $62,50 \%$ & $35,59 \%$ & $-26,91 \%$ \\
\hline Urbanidad & $62,50 \%$ & $38,98 \%$ & $-23,52 \%$ \\
\hline Simpatía & $37,50 \%$ & $49,15 \%$ & $11,65 \%$ \\
\hline Afabilidad & $37,50 \%$ & $37,29 \%$ & $-0,21 \%$ \\
\hline Cordialidad & $50,00 \%$ & $40,68 \%$ & $-9,32 \%$ \\
\hline Gratitud & $37,50 \%$ & $49,15 \%$ & $11,65 \%$ \\
\hline Indulgencia & $37,50 \%$ & $33,90 \%$ & $-3,60 \%$ \\
\hline Benignidad & $37,50 \%$ & $37,29 \%$ & $-0,21 \%$ \\
\hline Delicadeza & $37,50 \%$ & $35,59 \%$ & $-1,91 \%$ \\
\hline Respeto & $87,50 \%$ & $64,41 \%$ & $-23,09 \%$ \\
\hline Amistad & $75,00 \%$ & $57,63 \%$ & $-17,37 \%$ \\
\hline Cariño & $50,00 \%$ & $42,37 \%$ & $-7,63 \%$ \\
\hline Caridad & $37,50 \%$ & $47,46 \%$ & $9,96 \%$ \\
\hline Otras & $0,00 \%$ & $1,69 \%$ & $1,69 \%$ \\
\hline
\end{tabular}

En el curso 2009-2010, los profesores le daban mayor peso al desarrollo del autoconocimiento, empatía y perspectiva social, habilidades sociales y convivenciales, y a la cooperación (todos con un 87,50\%). En el curso 2010-2011, los porcentajes más altos corresponden a cooperar $(69,49 \%)$, que ha bajado respecto al año anterior $(-18,01 \%)$. En segundo lugar, se sitúa el respeto $(64,41 \%)$, y en tercero la amistad $(57,63 \%)$.

Llama la atención la excesiva variación negativa, de un curso académico a otro, de las siguientes habilidades: analizar necesidades $(-44,49 \%)$, empatía y perspectiva social ($34,96 \%)$, negociar y afrontar la complejidad $(-34,32 \%)$, y habilidades sociales y convivenciales $(-33,26 \%)$. Tener la capacidad de analizar necesidades, y negociar y afrontar la complejidad les permitirá, a los alumnos, a ser críticos y entender su entorno económico, político y social.

Una vez analizada la situación surgen muchos interrogantes. Uno de ellos es qué debemos hacer -y no qué podemos hacer- para formar en valores, en este contexto en 
crisis. Desde el ámbito educativo, creemos que hay que formar en base a auténticos criterios morales, que vayan más allá de la simple instrucción. Para ello, es importante trabajar competencias que desarrollen el sentido de la autonomía personal, la fuerza de la responsabilidad, la madurez espiritual y moral, y la solidaridad. En los tiempos que corren, caracterizados por la violencia, la educación en valores es una necesidad inexorable y un reto ineludible (González, 1995; Touriñán, 2008). Por tanto, en la educación, en el sentido estricto (Peiró, 2012a) debe haber un puesto privilegiado reservado a los valores morales, ya que estos contribuyen de forma decisiva a la formación integral del individuo.

\section{QUÉ Y CÓMO EDUCAR EN VALORES, EN TIEMPOS DE CRISIS}

Para entender qué es la educación en valores, debemos saber, primero, que la educación, en sí misma, ya es un valor. La educación en valores es el proceso mediante el cual se guía y se forma a los alumnos para que sean capaces de utilizar su experiencia axiológica de manera consciente para construir su proyecto personal de vida. Su objetivo es el desarrollo de destrezas, hábitos, actitudes y conocimientos, que capaciten a las personas para hacer, intervenir, comportarse, aprender e interrelacionarse con los demás, en base a valores.

Snook (2003) ya había señalado el nexo entre la teoría ética y la pedagogía en el aula. Este investigador argumenta que el maestro ético es el que comprende tanto el propósito moral de la educación y la importancia de considerar el proceso de enseñanza como esencialmente ética en su naturaleza.

La educación en valores implica la adquisición de un conjunto de competencias, que le capaciten al alumno para aprovechar las oportunidades y para dar respuesta a las exigencias de su entorno. Busca la formación integral del individuo, para que este se desenvuelva de manera competente en un entorno social, cultural y personal diverso (Touriñán, 2006a y 2006b). Por supuesto, es importante hacer énfasis en hecho de educarlos para que sepan aprovechar las oportunidades, desarrollarse y lograr sus objetivos, sin perjuicio de los demás, es decir, sin utilizar el conflicto ni la violencia, como instrumentos para ello (Díaz-Aguado, 1996). La educación en valores constituye un elemento fundamental para el autoconocimiento y para una óptima integración social y profesional del individuo. Esto implica "aprender a ser, aprender a respetar, aprender a valorar y aprender a aprender" (Moreno, Álvarez y Cardoso, 2001).

El hecho de cómo educar en valores y qué tipo de valores debemos transmitir, es una tarea compleja. Para dar respuesta a estas dos grandes interrogantes, debemos partir del análisis de la realidad global (externa al centro) y específica del aula. La realidad del contexto global nos muestra que se están acentuando una serie de problemas, que tienen un alcance universal, como son la pobreza, la desigualdad, la inseguridad, la intolerancia y últimamente, la corrupción generalizada, en las instituciones públicas y sociales. En este sentido, pese a que existe discrepancia en la universalización axiológica, es prioritario proponer la formación de unos valores éticos y morales universales, que no se basen en posturas doctrinarias específicas, sino en principios universales, no excluyentes. Asimismo, la realidad del aula es que la educación en valores no se trabaja como un eje transversal: se enseña valores, de manera esporádica y casual, y como un contenido más, los objetivos se reducen al contexto del aula, y la educación de valores personales y ciudadanos cada vez tiende a menos.

Intentado responder a la interrogante de cómo educar en valores en tiempos de crisis, encontramos las propuestas de Tierno (1992, 1993 y 1996) sobre los valores humanos, 
integrados con el modelo de De la Fuente (1999 y 2000), cuando afirman que los valores se deben trabajar en tres niveles: el nivel cognitivo, el nivel de los sentimientos y afectos, y el nivel de las acciones o comportamientos. Si la transmisión del conocimiento axiológico es imprescindible en la formación, es igualmente preciso el contacto directo con los valores y su vivencia.

De entre las diversas estrategias existentes para abordar la controversia social en las aulas (Morrison, et al. 2005), la indisciplina (Morrison, et al. 2001), etc., como son las que van desde buscar una mayor implicación del alumno en el proceso de enseñanza-aprendizaje, mejorar la comunicación y las relaciones interpersonales en el aula, plantear y desarrollar una disciplina asertiva, con plena autoridad del profesor, se subraya la tendencia a generar valores humanistas, teniendo en cuenta las características de los alumnos (Watson, et al. 1998).

Con el fin de definir más esto, se reconoce que el ejemplo vivido es mucho más edificante que las palabras. Ahora bien, el proceso educativo, que siempre es dinámico, naturalmente permite realizar cambios que modifican la pertinencia de los valores, de acuerdo con los intereses del educando. No obstante, el modelo más pertinente, con relación al alcance que la educación en valores debe tener en la estructura subjetiva del educando es el que se orienta al desarrollo integral del educando, al logro de la plenitud humana a través del desarrollo del juicio, del autocontrol y del ejercicio de una conducta clara y coherente (Peiró, 2012a) con los valores personales y sociales.

La base del acto didáctico se puede sintetizar en la realidad de la acción de cada alumno que, como especificación de la propia humana, no puede ser otra que "conocer-sintiendoconstruyendo" (Peiró, 2013, cap. 1). Este modelo, planteado por Peiró (1982) no busca una adquisición desordenada de valores, tampoco meros temas o conocimientos ideales, menos provocar sentimentalismos, sino una educación holista (Peiró, 2012b y 2013). La concepción de la educación axiológica funciona de la siguiente manera: el educando observa y conoce la realidad a través de la percepción (imitación, ejemplo, vicarianza). Este proceso se completa con la comprensión y el libre raciocinio-juicio del intelecto (estimativa). El segundo nivel implica la consecuente vida afectiva relativa al valor que se ha entendido (nadie ama lo que no conoce); se puede decir que es la inteligencia de los afectos, que da paso a las actitudes relativas a valores. La importancia de esta dimensión es crucial, ya que los procesos de aprendizaje, de todo tipo, incluido el aprendizaje de los valores, se energetizan mediante los sentimientos (pensemos en la levadura en su acción dentro de la masa de harina amasada). Por último, en esta educación axiológica holista, encontramos la dimensión del actuar de manera constructiva (Peiró, 1999, 2005, 2012b). ¿Qué se construye? Si se ha efectuado un juicio libre y los valores han sido estimados sin contaminar emocionalmente la inteligencia, las actitudes predisponen a que cada cual se formule propósitos que, si los realiza, serían acciones libres. La repetición de tal tipo de actos en libertad promueve en el escolar de unos hábitos positivos para cada tipo de valor. Entonces tenemos que uno va acumulando sus características: ordenado, puntual, esforzado, atento, estudioso, etc. El desarrollo de estas tres dimensiones, integradas y equilibradas daría como resultado una educación axiológica coherente y sólida... personalizada.

Con relación a los tipos de valores, no existe una clasificación única de los valores.

En síntesis, hablamos de la aplicación de un nuevo paradigma axiológico que se separe del racionalismo mecanicista de una educación axiológica holística (del conjunto y no sólo de cada parte), sistemática y organizada, y que sea interdisciplinaria y transversal a todo el currículo educativo. 


\section{CONCLUSIONES E IMPLICACIONES}

El sistema educativo es un medio importante para contener los efectos de la transformación de la crisis económica en crisis moral, razón más que suficiente para impedir cualquier acción que contravenga la formación integral del educando, que no mantenga la calidad educativa o, en definitiva, que disminuya la cantidad y la calidad de los recursos asignados. Si bien se ha avanzado, en el ámbito educativo, aún queda mucho por hacer y en condiciones poco fáciles. Con los recortes en educación, estamos entrando en un proceso de destrucción de los principios sociales, morales y éticos.

La educación en valores es un elemento fundamental para el autoconocimiento y para la integración social y profesional. Les permitirá, a los alumnos, aprender a ser, aprender a respetar, aprender a valorar y aprender a aprender. Por tanto, la escuela debe ser el contexto formal, desde donde se promueva la integración del desarrollo personal, la integración ciudadana y la tolerancia y solidaridad, en un mundo diverso, y al mismo tiempo global. Los valores se pueden aprender en contextos informales y formales, especialmente en la familia y en el contexto escolar. Se pueden aprender o desaprender. En su aprendizaje, el sujeto puede desempeñar un papel activo, o, por el contrario, puede adoptar valores de manera inconsciente (Hernández, 2002). Por ello, creemos que es importante organizar y tomar decisiones previas sobre qué, para qué y cómo se puede trabajar la educación en valores en el aula.

Los valores se deben trabajar en tres niveles: el nivel cognitivo, el nivel de los sentimientos y afectos, y el nivel de las acciones o comportamientos. La transmisión del conocimiento axiológico es imprescindible en la formación, pero lo es también su vivencia.

Por otro lado, este estudio nos permite concluir que los profesores no realizan un trabajo sistemático de educación en valores por falta de tiempo y por falta de propuestas de actividades concretas. Hay una escasa coordinación entre ellos. Esto puede estar relacionado con la inexistencia, desconocimiento o falta de efectividad de un Programa de Educación en Valores, que se refleje en el proyecto educativo, en el proyecto curricular del centro, y, en concreto, en los distintos proyectos de actuación pedagógica del centro (Plan de Convivencia, Plan de Acción Tutorial, Plan de Atención a la Diversidad, etc.).

Lo que más les preocupa a los profesores es conseguir una mayor disciplina y orden para trabajar y mejorar el cumplimiento de normas en el aula, cuando la educación en valores debe trascender al aula, y debe servir para mejorar la convivencia social.

El desarrollo de habilidades sociales es el ítem que más ha variado $(-39,41 \%)$ negativamente. Esto nos indica que es crucial trabajar con los estudiantes el desarrollo de estas habilidades, desde su formación inicial. Hace falta un trabajo permanente y continuado de educación en valores, desde todas las asignaturas, y no sólo en las horas destinadas a la tutoría.

Evidentemente, la educación en valores nos presenta varios desafíos: considerar la educación en valores como un elemento natural del proceso enseñanza-aprendizaje; formar en valores universales, pero también en valores determinados por la cultura a la que pertenecen los alumnos, porque no se puede enseñar en un vacío cultural; construir la escuela como un modelo de práctica democrática, que permita a los alumnos entender, a partir de problemas concretos, cuáles son sus derechos y deberes y cómo el ejercicio de su libertad está limitado por los derechos y la libertad de los demás, y potenciar la formación, en base a una axiología educativa, para formar individuos y ciudadanos competentes. 


\section{BIBLIOGRAFÍA}

BEAR, G. et al. (2000), "Prevention of school violence", in G. Bear y C. Minke (eds.) Preventing school problems-Promoting school success: Strategies that work, Washington, DC, National Association of School Psychologists, pp. 1-69.

BENBENISHTY, R. y ASTOR, R. (2005), School violence in context: culture, neighborhood, family, school and gender, New York, Oxford.

DE LA FUENTE, J. (1999), Educación para la construcción personal. Un enfoque de autorregulación en la formación de profesores y alumnos, Bilbao, Desclée de Brouwer.

DE LA FUENTE, J. (2000), "Taller de mejora personal: aprendemos a regular nuestros pensamientos, sentimientos y acciones", en M. Álvarez y R. Bisquerra Manual de Orientación y Tutoría, Barcelona, Ciss Praxis, 330/225-330/239.

DÍAZ-AGUADO, M. J. (1996), Programa de educación para la tolerancia y prevención de la violencia en los jóvenes. Madrid, Instituto de la Juventud. Ministerio de Trabajo y Asuntos Sociales. GONZÁLEZ, R. (1995), "Disciplina y control en la clase”, en J.A. Beltrán y A. Bueno (eds.), Psicología de la Educación, Barcelona, Marcombo.

HERNÁNDEZ, P. (2002), Los moldes de la mente, La Laguna, Tafor.

LEY ORGÁNICA DE EDUCACIÓN (LOE, 2006), BOE nº 106, de 4 de mayo de 2006.

LOVAT, T. (2009), "Values Education and Quality Teaching. Two Sides of the Learning Coin", in LOVAT, T. y RON, T. (ed.), Valius Education and Quality Teaching. The Double Helix Effect, New York, Springer, 1-12.

MERMA, G. (2011), "How do teachers deal with values education in schools? The case of Valencia Community", European Conference on Educational Research, Berlín, Freie Universität Berlin.

MERMA, G. (2012), "Análisis de los componentes axiológicos en la legislación educativa española y autonómica: el caso de la Comunidad Valenciana", en S. Peiró (coord.), Convivencia en educación: problemas y soluciones. Perspectivas Europea y Latinoamericana, Alicante, Universidad de Alicante, pp. 63-82.

MORENO, M. T.; ÁLVAREZ, N. y CARDOSO, R. (2001), "Hacia una Pedagogía de los valores de la Educación Superior", Tendencias pedagógicas, 6, pp. 39-54.

MORRISON, G. M. et al. (2001), "School expulsion as a process and as event: Before and after effects on children at-risk for school discipline", in R.J. Skiba, y G.G. Noam (eds.), New directions in youth development-Zero tolerance: Can suspension and expulsion keep schools safe? 92, pp. 45-72.

MORRISON, G. M. et al. (2005), "The safe school: Integrating the School Reform Agenda to Prevent Disruption and Violence at School", in A. Goldstein, y J.C. Conley (eds.), School Violence Intervention: A Practical Handbook, New York, Guilford, pp. 256-297.

MUÑOZ, R. F.; MRAZEK, P. y HAGGERTY, R. (1996), "Institute of Medicine Report on Prevention of Mental Disorders: Summary and commentary", American Psychologist, $\mathrm{n}^{\circ}$ 51, pp. 1116-1122.

OSHER, D., DWYER, K. y JACKSON, S. (2004), Safe, supportive and successful schools step by step, Longmont, CO, Sopris West.

PEIRÓ, S. (1982), El ideario educativo: axiología e interdisciplinaridad, Madrid, Narcea.

PEIRÓ, S. (1999), Modelos teoréticos en educación. Interpretación antropológica, Alicante, ECU.

PEIRÓ, S. (2005), Indisciplina y violencia escolar, Alicante, Instituto Alicantino de Cultura.

PEIRÓ, S. (2009), Valores educativos y convivencia, España, Club Universitario.

PEIRÓ, S. (2012), "Indisciplina, contexto institucional y docencia", en Peiró, S. (2012) Convivencia en educación: problemas y soluciones. Perspectivas europea y latinoamericana (coord.), Alicante, Universidad de Alicante, pp. 33-62.

PEIRÓ, S. (2013) (dir.), Modelos de educar en valores, Madrid, Dykinson.

PEIRÓ, S. y BERESALUCE, R. (2012), "Subjetividad y educabilidad. Orientaciones para la praxis docente", Exedra n ${ }^{\circ} 6$, pp.105-122.

RODRÍGUEZ, R. L. y LUCA DE TENA, C. (2001), Programa de Disciplina en la Enseñanza Secundaria Obligatoria ¿cómo puedo mejorar la gestión y el control de mi aula? Málaga, Aljibe.

SNOOK, I. (2003), The ethical teacher, Palmerston North, NZ, Dunmore. 
TIERNO, B. (1992), Valores humanos II, Madrid, Taller de Editores S. A.

TIERNO, B. (1993), Valores humanos I y III, Madrid, Taller de Editores S. A.

TIERNO, B. (1996), Guía para educar en los valores humanos, Madrid, Taller Editores S. A.

TOURIÑÁN, J. M. (dir.) (2008), Educación en valores. Educación intercultural y formación para la convivencia pacífica, España, Netbiblo.

TOURIÑÁN, J. M. (2006a), "Conocer, estimar, enseñar, elegir y realizar valores: retos epistemológicos de la investigación pedagógica", Universitas Tarraconensis Revista de Ciencies de l'educació, pp. 173-212.

TOURIÑÁN, J. M. (2006b), "Educación en valores y experiencia axiológica: el sentido patrimonial de la educación", Revista Española de Pedagogía, nº 64, pp. 227-248.

WATSON, M. (1998), Developmental discipline, en: http://tigger.uic.edu/ Inucci/MoralEd/practices /practice2watson.html

\section{Breve currículo}

\section{Gladys Merma Molina}

Doctora por la Universidad de Alicante. Profesora ayudante doctora en el Departamento de Didáctica General y Didácticas Específicas. Máster en Comunicación y Cultura, Máster en Educación y Tecnologías de la Información y de la Comunicación, Máster en Administración, y especialista en Dirección de Organizaciones Públicas. Investigación: entre otros, Proyecto de Redes de Investigación en Docencia Universitaria y el Proyecto de pedagogía GITE 09036UA: Grupo de Innovación Tecnológico-Educativa de la Universidad de Alicante. Diversos libros y artículos sobre el contacto lingüístico peruano. Coordinadora de: Aportaciones curriculares para la interacción en el aprendizaje. Artículos y capítulos de libros sobre la educación y la filología. Comunicaciones en congresos nacionales e internacionales. Es coordinadora académica de III Programa de Postgrado en Gestión y Dirección Pública, Universidad de Alicante.

\section{Salvador Peiró i Gregòri}

Profesor titular, Facultad de Educación, Universidad de Alicante. Profesor de EGB (1968-1979). Docencia en la Universidad de Valencia (1978-1983), colaborador de la Cátedra de Pedagogía General. Investigaciones de postgrado sobre interdisciplinaridad y valores en educación. Profesor Universidad de Granada (1973/4-1996/7), donde organiza el grupo de investigación interdisciplinar sobre teoría de la educación social en materia de violencia, riesgo y marginación. Desde 1997/98 en la Universidad de Alicante. Dirige un equipo de investigación sobre indisciplina, valores y violencia en educación. Ha publicado unos 150 libros y artículos, fundado asociaciones científicas, ha sido Director del Departamento de Pedagogía, y de la Revista de Educación de la Universidad de Granada y es miembro del consejo de redacción de varias revistas. En todas las universidades, ha organizado, gestionado y dirigido cursos de postgrado, congresos, jornadas, etc.

\section{Diego Gavilán Martín}

Profesor colaborador. Facultad de Educación de la Universidad de Alicante. Actualmente imparte asignaturas del Área de Teoría e Historia de la Educación y otras vinculadas con el uso de la TIC en la educación. Ha elaborado el sitio Web del Grupo Interdisciplinar de Docencia Universitaria (GIDU2008) y ha desarrollado el sitio Web del Proyecto Diseño y Atención a las Oportunidades de Género en la Educación Superior (2012), de la Universidad de Alicante. Es un profesional del campo de la comunicación digital y corporativa y el diseño gráfico, Web y multimedia. Es fundador del Estudio de Diseño y Publicidad TAIMARA. 